\title{
Identification of a pathogenic mutation in a Chinese pedigree with polycystic kidney disease
}

\author{
KEXIAN DONG $^{1 *}$, HUANHUAN MIAO $^{2 *}$, XUEYUAN JIA $^{1}$, JIE WU $^{1}$, HAN WU $^{2}$, JIAWEI SUN ${ }^{2}$, \\ WEI JI ${ }^{1}$, HUI SU ${ }^{1}$, LIDAN XU ${ }^{1}$, XUELONG ZHANG ${ }^{1}$, SIQI ZHU ${ }^{1}$, GUOHUA JI ${ }^{1}$, RONGWEI GUAN ${ }^{1}$, \\ HAO WANG $^{3}$, JING BAI ${ }^{1}$, JINGCUI YU ${ }^{4}$, WENJING SUN ${ }^{1}$, XIANLI ZHOU ${ }^{2}$ and SONGBIN FU ${ }^{1}$ \\ ${ }^{1}$ Laboratory of Medical Genetics, Harbin Medical University, Harbin, Heilongiiang 150081; \\ ${ }^{2}$ In-Patient Ultrasound Department, ${ }^{3}$ Department of Hepatopancreatobiliary Surgery, ${ }^{4}$ Scientific Research Centre, \\ The Second Affiliated Hospital of Harbin Medical University, Harbin, Heilongjiang 150086, P.R. China
}

Received May 25, 2018; Accepted December 18, 2018

DOI: $10.3892 / \mathrm{mmr} .2019 .9921$

\begin{abstract}
Polycystic kidney disease (PKD) is a life-threatening inherited disease with a morbidity of 1:500-1,000 worldwide. Numerous progressively enlarging cysts are observed in the bilateral kidneys of patients with PKD, inducing structural damage and loss of kidney function. The present study analyzed one family with PKD. Whole exome sequencing of the proband was performed to detect the pathogenic gene present in the family. Candidate gene segments for lineal consanguinity in the family were amplified by nest polymerase chain reaction, followed by Sanger sequencing. One novel duplication variant (NM_001009944.2:c.9359dupA:p.Y3120_E3121delinsX) and one missense mutation (c.G9022A:p.V3008M) were detected in PKD1. Additionally, the pathogenic substitutions in PKD1 published from the dataset were analyzed. Following analysis and confirmation, the duplication variant NM_001009944.2:c.9359dupA:p.Y3120_E3121delinsX in PKD1, within the polycystin-1, lipoxygenase, $\alpha$-toxin domain, was considered to be the pathogenic factor in the examined family with autosomal dominant PKD. Additionally, based
\end{abstract}

Correspondence to: Professor Songbin Fu, Laboratory of Medical Genetics, Harbin Medical University, 157 Baojian Road, Nangang, Harbin, Heilongjiang 150081, P.R. China

E-mail: fusb@ems.hrbmu.edu.cn

Professor Xianli Zhou, In-Patient Ultrasound Department, The Second Affiliated Hospital of Harbin Medical University, 246 Xuefu Road, Nangang, Harbin, Heilongjiang 150086, P.R. China

E-mail: hrbzhouxl@163.com

*Contributed equally

Abbreviations: PKD, polycystic kidney disease; ADPKD, autosomal dominant polycystic kidney disease; ARPKD, autosomal recessive polycystic kidney disease; PC1, polycystin-1; PC2, polycystin-2; ESRD, end-stage renal disease; LR-PCR, long-range polymerase chain reaction

Key words: ADPKD, PKD1, duplication variant, pedigree, PC1 on the analysis of 4,805 pathogenic substitutions in PKD1 within various regions, the presence of the missense mutation in the N-terminal domain of polycystin-1 may present high pathogenicity in ADPKD.

\section{Introduction}

Polycystic kidney disease (PKD) is a monogenic inherited disease characterized by bilateral polycystic kidneys and their progressive enlargement, which affects renal structure and function (1). According to the inheritance pattern, PKD is divided into autosomal dominant PKD (ADPKD, OMIM\#173900) and autosomal recessive PKD (ARPKD, OMIM\#263200). ADPKD is a life-threatening disease, and is one of the most common inherited diseases, with a morbidity of 1:500-1,000, affecting >12 million individuals worldwide (2-4). In addition to the kidneys, other organs may also be affected, including the liver, brain and pancreas, resulting in end-stage kidney disease (ESRD) (5-7). The manifestations of ADPKD usually appear in adulthood; the majority of individuals with ADPKD have hypertension (8-10). There are two primary genes in which mutations are reported to lead to ADPKD: PKD1 (OMIM*601313, autosomal dominant) and PKD2 (OMIM*173910, autosomal dominant). PKD1 is located on chromosome 16p13 (11). The protein product of PKD1, polycystin-1 (PC1), is an integral membrane protein with 11 membrane-spanning domains. PKD2 is located on chromosome 4q22.1, and its protein product, polycystin-2 (PC2), also localizes to the cell membrane, acting as a nonselective $\mathrm{Ca}^{2+}$ channel (12). The C-termini of PC1 and PC2 interact to regulate ion transportation; thus, mutations in PKD1 and PKD2 may lead to disease (13-16).

Over 2,000 and 400 mutations in PKD1 and PKD2, respectively, have been identified in association with PKD; 85\% of patients with ADPKD carry mutations in PKD1 and its protein product PC1, which comprises numerous domains (17). The PC1 C-terminal domains are the most extensively studied, and include a coiled-coil domain (amino acids 4,220-4,251) and a G-protein binding and activation sequence (amino acids 4,111-4,184) (18). The coiled-coil domain mediates PC2 binding in order to control $\mathrm{Ca}^{2+}$ flow (19). Providing the PC1/PC2 
complex loses its function, intracellular $\mathrm{Ca}^{2+}$ levels become too low to activate phosphatidylinositol 3-kinase or Akt, which activate Camp-dependent B-Raf $(20,21)$. Thus, cyclic adenosine monophosphate (cAMP) induces the activation of the mitogen-activated protein kinase/extracellular signal-regulated kinase pathway via protein kinase A/Ras/B-Raf signaling, stimulating cell proliferation and promoting the expansion of cystic cavities (21-23). Due to cAMP stimulation, cystic epithelial cells secrete $\mathrm{Cl}^{-}$together with $\mathrm{Na}^{+}$via the paracellular pathway $(21,24)$. Additionally, the $\mathrm{C}$-terminal tail of $\mathrm{PC} 1$ interacts with the $\alpha$-subunit of $\mathrm{Na}^{+} / \mathrm{K}^{+}$-adenosine 5 '-triphosphatase, increasing $\mathrm{Na}^{+}$ and $\mathrm{K}^{+}$translocation (25). During osmotic action, fluid is extracellularly secreted and accumulates in cystic cavities (25); as a result, the kidneys progressively engorge and fill with fluid. The G-protein-coupled receptor (GPCR) phosphorylation site (GPS) motif within PC1 is part of the GPCR-autoproteolysis inducing regulatory domain, which mediates $\mathrm{PC1}$ cis-autoproteolysis to generate an unglycosylated $\mathrm{N}$-terminus and a $\mathrm{C}$-terminus that remains non-covalently bound $(19,26)$. The function of the observed cleavage remains unknown; however, it may aid stable protein folding and PC1 trafficking (27).

In the present study, whole exome sequencing of a blood sample from a proband with ADPKD was performed to detect the pathogenic variations present within their family. To identify the pathogenic mutation site, nested polymerase chain reaction (PCR) was conducted to amplify candidate regions present in the patient and other unaffected family members. The PCR product was sequenced via Sanger sequencing. As a result, the novel mutation was determined to be the pathogenic factor. The mutation generates a truncated PC1 without its C-terminal domains. Additionally, all the pathogenic substitutions in PKD1 published from the dataset were analyzed in order to evaluate the pathogenic domains of PC1, representing the first report of this nature, to the best of our knowledge. The present study provides a basis for assessing the pathogenic properties of missense mutations, and may aid in the genetic counseling of patients with ADPKD, and contribute to the identification of asymptomatic individuals at risk of developing ADPKD.

\section{Materials and methods}

Subjects and blood samples. The present study was approved by the Institutional Research Board of Harbin Medical University (Harbin, China), and all participants provided written informed consent. A four-generation family was recruited on May, 2017; the pedigree of the family included 29 individuals with 15 females and 14 males. Clinical information and peripheral blood samples from the proband and their daughters were collected into graded negative pressure vacuum EDTA anticoagulant tubes.

Clinical evaluation. The family with ADPKD was identified at The Second Affiliated Hospital of Harbin Medical University (Harbin, China). The proband was a 56-year-old female who had been diagnosed with PKD and polycystic liver disease one year prior to analysis. Ultrasound examination for abdominal urology assessment revealed numerous cysts of variable sizes in the patient's bilateral kidneys and liver. The kidneys and liver were notably enlarged. The largest cyst in the liver was $\sim 72 \times 58 \mathrm{~mm}$. The size of the cholecyst was normal; however, the gallbladder wall was thick and rough. Additionally, the pancreas was normal. Liver puncture was performed to extract the cystic liquid following local anesthetization with $2 \%$ lidocaine; $430 \mathrm{ml}$ of cystic liquid was extracted twice within one week from 16 cysts.

Pathogenic gene detection. Whole exome sequencing of the blood sample from the proband was performed by Novogene Co., Ltd. (Beijing, China). For library generation, $1.0 \mu \mathrm{g}$ of genomic DNA per sample was required, which was randomly fragmented to $180-280$ base pairs (bp) by a sonicator (Covaris S220, Covaris, Woburn, MA, USA) using $450 \mathrm{~W}$ peak incident power at 20 cycles per burst for $400 \mathrm{sec}$. The DNA fragments were then end-repaired and phosphorylated (Illumina, Inc., San Diego, CA, USA). Following determination of the size distribution and concentration of the fragments, the DNA library was sequenced on the Illumina HiSeq 4000 platform for paired-end 150 bp reads. DNA extraction was performed using the DNeasy Blood and Tissue kit (Qiagen GmbH, Hilden, Germany) according to the manufacturer's protocols. Briefly, a DNA collection spin tube was employed to isolate genomic DNA from the blood sample. As the PKD1 gene is complex, long-range PCR (LR-PCR) was performed to amplify PKD1 exons 22-26 (forward, 5'-TCCAGTCAAGTG GGCTCTC-3', and reverse, 5'-CAATGAAGAGGAAAGCAG CAC-3'). In the LR-PCR system, $100 \mathrm{ng}$ template, $2 \mu \mathrm{ldNTP}$ (4019; Takara Bio, Inc., Otsu, Japan), $10 \mu 1$ 2XGC buffer (9155; Takara Bio, Inc.), $1 \mu \mathrm{l}$ forward primer (10 pmol/l), $1 \mu \mathrm{l}$ reverse primer (10 pmol/l), $0.2 \mu \mathrm{l}$ LA Polymerase (RR02MA; Takara Bio, Inc.) and water were used: $20 \mu \mathrm{l}$ in total. The thermocycling condition followed the LA Polymerase protocol: Initialization at $94^{\circ} \mathrm{C}$ for $1 \mathrm{~min}$, denaturation at $98^{\circ} \mathrm{C}$ for $10 \mathrm{sec}$, annealing at $63^{\circ} \mathrm{C}$ for $30 \mathrm{sec}$, extension at $72^{\circ} \mathrm{C}$ for $3.5 \mathrm{~min}$, a final elongation at $72^{\circ} \mathrm{C}$ for $10 \mathrm{~min}$ and hold at $4^{\circ} \mathrm{C}$, for 30 cycles. The LR-PCR product was amplified as the template to obtain the candidate region within the 26th exon (forward, 5'-GGCTGCCCACCCTGACTG ACT-3', and reverse, 5'-CCTGGGTGGGCTCGGCTCTATC-3') and the 25th exon (forward, 5'-CGCCGGGCCCAGGAGGTC-3', and reverse, 5'-CCCCGGCCCAATGAGCACAA-3'). In the PCR system, $100 \mathrm{ng}$ template, $3 \mu \mathrm{l} \mathrm{dNTP}$ (4019, Takara, Japan), $3 \mu \mathrm{l}$ 10XGC buffer (9151A; Takara Bio, Inc.), $1.5 \mu \mathrm{l}$ forward primer (10 pmol/l), $1.5 \mu \mathrm{l}$ reverse primer (10 pmol/1), $0.3 \mu \mathrm{l}$ Taq Polymerase (R001A; Takara Bio, Inc.) and water were used: $30 \mu \mathrm{l}$ in total. The thermocycling condition followed the Taq Polymerase protocol: Initialization at $98^{\circ} \mathrm{C}$ for $1 \mathrm{~min}$, denaturation at $98^{\circ} \mathrm{C}$ for $10 \mathrm{sec}$, annealing at $61^{\circ} \mathrm{C}$ for $30 \mathrm{sec}$, extension at $72^{\circ} \mathrm{C}$ for $45 \mathrm{sec}$, final elongation at $72^{\circ} \mathrm{C}$ for $10 \mathrm{~min}$ and hold at $4^{\circ} \mathrm{C}$, for 30 cycles. The nest PCR product was ligated to the $\mathrm{T}$ linear vector (Peasy-T1 Simple Cloning Kit, cat. no. CT111, Beijing Transgen Biotech Co., Ltd., Beijing, China) according to the manufacturer's protocol and sequenced by TsingKe Biological Technology (Beijing, China) via Sanger sequencing.

Variation analysis. During whole exome sequencing, the reads that aligned to exonic regions were collected for variant calling, and the identification of single nucleotide polymorphisms (SNPs) and indels using SAMtools mpileup and bcftools (28). The frequency of variants was evaluated via the 1000 Genomes Project (http://browser.1000genomes.org) and the Exome Aggregation Consortium (http://exac.broadinstitute.org). The 
significance of mutation variants was assessed with the Online Mendelian Inheritance in Man database (OMIM, http://omim. org; clinical significance is assessed as pathogenic), the Human Gene Mutation Database (HGMD, http://www.hgmd.cf.ac. uk/ac/all.php; clinical significance is assessed as pathogenic), the SNP Database (dbSNP; https://www.ncbi.nlm.nih. gov/projects/SNP/snp_ref.cgi?geneId=5310; clinical significance is assessed as pathogenic) and the Autosomal Dominant Polycystic Kidney Disease Database (PKDB, http://pkdb.pkdcure. org; clinical significance is assessed as any pathogenic mutation), as well as the substitution assessment tools SIFT (https://sift. bii.a-star.edu.sg; score $\leq 0.05$ ), Polyphen2_HVAR (http:/genetics. bwh.harvard.edu/pph2; score $\sim 1$ assessed as probably pathogenic or possibly pathogenic), MutationTaster (http://www.mutationtaster.org; small score assessed as disease-causing automatic or disease-causing) and Mutation Assessor (http://mutationassessor. org/r3/; large score assessed as high or medium). The conserved properties of the variants were analyzed using the University of California Santa Cruz Genome Browser (http://genome.ucsc.edu). The division of variable regions in PC1 was based on UniProt (http://www.uniprot.org).

\section{Results}

Studied family suffers from ADPKD. A family with ADPKD, including 29 individuals from four generations, was studied. The family's medical history was further investigated following diagnosis of the proband with PKD. The proband was a 56-year-old female who was diagnosed with bilateral PKD and polycystic liver disease at the age of 55. After one year, the proband returned for a check-up and treatment. According to the results of an abdominal ultrasound examination (Table I), numerous cysts in the liver were filled with cystic liquid; thus, liver puncture treatment was required.

Based on the pedigree (Fig. 1), five patients with PKD were evaluated in the family, including two females and three males. The proband's father was diagnosed with PKD, while their mother exhibited no symptoms. The remaining diagnosed patients in the family were sisters or brothers of the proband; however, the two daughters of the proband, who were 34 and 27 years old, exhibited no manifestations of PKD. The other individuals in generations III and IV were not examined or diagnosed. As the clinical symptoms of the disease are not clear, it is difficult to determine whether individuals suffer from the disease or not without ultrasound examination. Almost half of the lineal consanguineous group (5 of 11 individuals) was diagnosed with PKD. Collectively, the results indicated that the family analyzed suffers from ADPKD.

A novel duplication variant,NM_001009944.2:c.9359dupA:p. Y3120_E3121delinsX, may be the pathogenic mutation. To identify the pathogenic genes associated with PKD, whole exome sequencing was performed on the proband. The results revealed an adenine (A) duplication following chr16:2152099 (GRCh37/hg19, GCF_000001405.25), which resulted in a frameshift mutation in PKD1 and generated a truncated PC1 protein. The inserted A was located in the 26th exon of PKD1 in a heterozygous form. The duplication variant was described as c.9359dupA:p.Y3120_E3121delinsX in version NM_001009944.2. Additionally, one missense
Table I. Abdominal ultrasound examination findings.

\begin{tabular}{lccc}
\hline Individuals & $\begin{array}{c}\text { Age } \\
\text { (years })\end{array}$ & Sex & Findings \\
\hline $\mathrm{II}_{4}$ & 56 & Female & $\begin{array}{l}\text { Polycystic kidney and } \\
\text { polycystic liver with large } \\
\text { liver, chronic cholecystitis }\end{array}$ \\
$\mathrm{III}_{5}$ & 34 & Female & $\begin{array}{l}\text { Non-apparent abnormality } \\
\text { Non-apparent abnormality }\end{array}$ \\
$\mathrm{III}_{6}$ & 27 & Female & Non-appority
\end{tabular}

site (NM_001009944.2:c.G9022A:p.V3008M) and two synonymous sites (NM_001009944.2:c.G4674A:p.T1558T and NM_001009944.2:c.G3444A:p.P1148P) were observed in PKD1 (Table II, Fig. S1).

Notably, the duplication variant (c.9359dupA:p.Y3120_ E3121delinsX) identified in PKD1 is a novel mutation that has not been reported in PKDB, OMIM, HGMD or other published articles. To determine the mutations present in the family, targeted DNA fragments from the proband and their two normal daughters were sequenced via Sanger's method and analyzed. The results of Sanger sequencing indicated that the mother possessed the duplication variant c.9359dupA:p. Y3120_E3121delinsX in PKD1, while their two normal daughters did not (Fig. 2A and C). Further investigation of the proband's sequence was performed via ligation of the nest PCR product with the $\mathrm{T}$ linear vector, followed by sequencing. The sequencing results revealed that the proband carried one mutated sequence (Fig. 2D) and one normal sequence (Fig. 2E), indicating that the mutation exists in a heterozygous form.

In addition, the proband and their daughters presented the same c.G9022A:p.V3008M mutation in PKD1 (Fig. 3A-C). To further investigate the importance of the missense site, the pathogenic and conservational properties of the site were analyzed using various prediction tools. Almost all the prediction results indicated that the missense site was pathogenic (Table III). The Polyphen2 prediction results for c.G9022A:p. V3008M are presented in Fig. 3D. Conservational property analysis revealed that human, mouse and other animals, but not chicken, share the same amino acid (valine, V) at a particular site, indicating high conservation (Fig. 3E). PC1 is an integral membrane protein comprising 4,303 amino acids; $>3,000$ of the amino acids in the first part of the protein are located extracellularly. A diagram of PC1 is presented in Fig. 4. The position of c.9359dupA:p.Y3120_E3121delinsX is located within the PC-1, lipoxygenase, $\alpha$-toxin (PLAT) domain, and c.G9022A:p.V3008M is located within an undefined region (amino acids 2,834-3,011) near the GPS domain (Fig. 4). The first 23 amino acids in PC1 consist of a signal peptide that is cleaved during the post-translational modification process (29). The remaining PC1 protein can be separated into extracellular, transmembrane and cytoplasmic regions that contain numerous domains.

Analysis of all the pathogenic sites of PKD1 suggests that the missense c.G9022A:p.V3008M mutation is likely pathogenic. The PKDB is a database that facilitates the characterization of ADPKD variants in PKD1 and PKD2. In PKDB, mutations 
Table II. PKD1 variant information.

\begin{tabular}{llllllll}
\hline Exon & Variant & Genotype & \multicolumn{1}{c}{ NM number } & \multicolumn{1}{c}{ Amino acid change } & Domain & $1000 \mathrm{G}^{\mathrm{a}}$ & ExAC $^{\mathrm{b}}$ \\
\hline 26 & Duplication & Heterozygote & NM_001009944.2 & c.9359dupA:p.Y3120_ & PLAT & - & - \\
& & & & E3121delinsX & & \\
25 & Missense & Heterozygote & NM_001009944.2 & c.G9022A:p.V3008M & Near GPS & 0.001597 & 0.0005109 \\
15 & Synonymous & Heterozygote & NM_001009944.2 & c.G4674A:p.T1558T & PKD11 & 0.07109 & 0.07574 \\
15 & Synonymous & Heterozygote & NM_001009944.2 & c.G3444A:p.P1148P & PKD6 & 0.003395 & 0.003747 \\
\hline
\end{tabular}

${ }^{a}$ Frequencies listed in the 1000 Genomes Project $(1000 \mathrm{G}$ ) among all public databases in August 2015. A frequency $<0.01$ is considered meaningful. ${ }^{b}$ Frequencies listed in the Exome Aggregation Consortium (ExAC) database among all public databases in August 2016. A frequency $<0.01$ is considered meaningful. GPS, G protein-coupled receptor proteolysis site; PKD, polycystic kidney disease; PLAT, polycystin-1, lipoxygenase, $\alpha$-toxin.

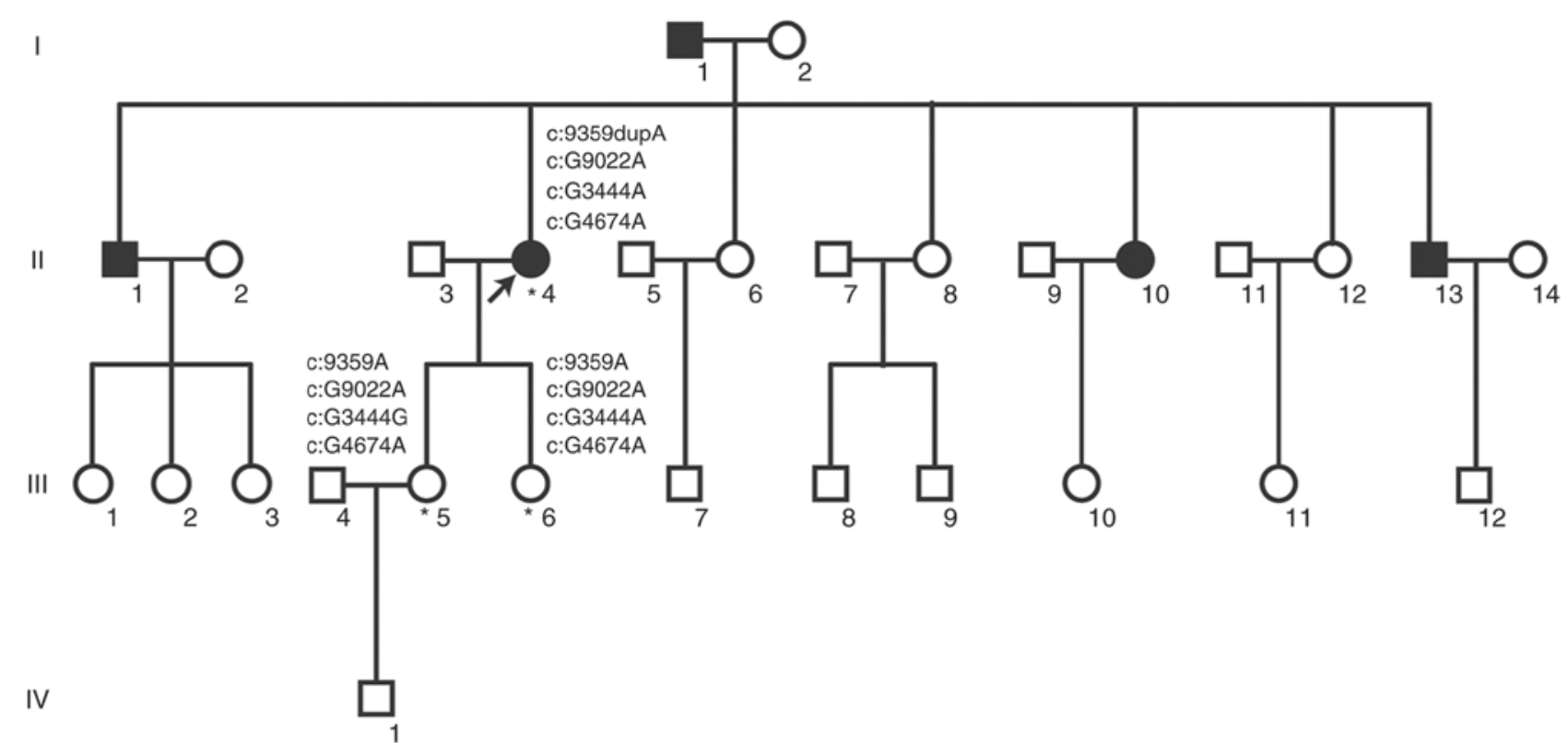

Figure 1. Pedigree of the studied family. Squares represent males, and circles represent females. Black indicates patients. The arrow indicates the proband. Asterisks indicate the individuals that were clinically examined and underwent genetic analysis.

in PKD1 and PKD2 are collected, and ADPKD mutation types are classified as substitution, nonsense, synonymous, 3'-untranslated region (UTR), 5'-UTR, frameshift, insertion or deletion, large deletion or duplication, splice, intervening sequence (IVS) silent and IVS unknown mutations. The clinical significance of each mutation is further characterized as definitely pathogenic, highly likely pathogenic, likely pathogenic, indeterminate, likely hypomorphic or likely neutral (30). According to the PKDB, it is difficult to identify the clinical significance of substitution mutations, and no substitutions have been classified as definitely pathogenic.

To further investigate the pathogenic properties of the single nucleotide variants in various regions, PKD1 substitutions listed in dbSNP and PKDB were studied. The single nucleotide variants from the two websites were counted, and repetitions were deleted. As the clinical significance of nonsense mutations is assessed as definitely pathogenic, the single nucleotide variant group in dbSNP was excluded. Substitutions described as likely pathogenic and highly likely pathogenic in PKDB were counted. In total, 4,805 single nucleotide variants, including 312 pathogenic variants, were observed in PC1 amino acids 24-4,303.

Provided that PC1 is an integral membrane protein, in addition to its signal peptide, it can be divided into an extracellular region (amino acids 24-3,074), a transmembrane region (amino acids 3,075-4,106) and a cytoplasmic region (amino acids 4,107-4,303). The frequency of substitutions identified as highly likely pathogenic or likely pathogenic was calculated within a variety of regions with various numbers of single nucleotide variants. Notably, the frequencies of highly likely pathogenic or likely pathogenic substitutions in the extracellular $(6.3 \%, 216$ out of 3,444 amino acids) and transmembrane ( $7.9 \%, 88$ out of 1,111 amino acids) regions were similar, while that in the cytoplasmic region was markedly lower $(3.2 \%$, 8 out of 250 amino acids) (Table III). The results suggested that substitutions occurring in the $\mathrm{PC} 1 \mathrm{~N}$-terminal region were more likely to be pathogenic variants than substitutions in the C-terminal region of the protein. 
Table III. Assessment of the missense site in PKD1.

\begin{tabular}{lcccc}
\hline Mutation & SIFT $^{\mathrm{a}}$ & Polyphen2_HVAR $^{\mathrm{b}}$ & MutationTaster $^{\mathrm{c}}$ & MutationAssessor $^{\mathrm{d}}$ \\
\hline c.G9022A:p.V3008M & 0.005 & 0.982 & $21, \mathrm{D}$ & $2.865, \mathrm{M}$ \\
\hline
\end{tabular}

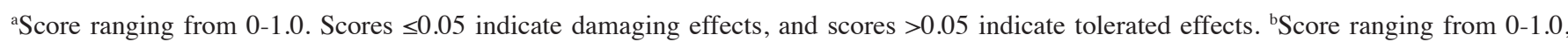
where 1.0 is more damaging. 'S core ranging from 0-215, where a smaller score is more different than the original amino acid. The result can be A (disease-causing automatic), D (disease-causing), $\mathrm{N}$ (polymorphism) or P (polymorphism automatic). If the score is larger and more pathogenic, the result indicates a change in function as $\mathrm{H}$ (high), $\mathrm{M}$ (medium), L (low) or N (neutral).

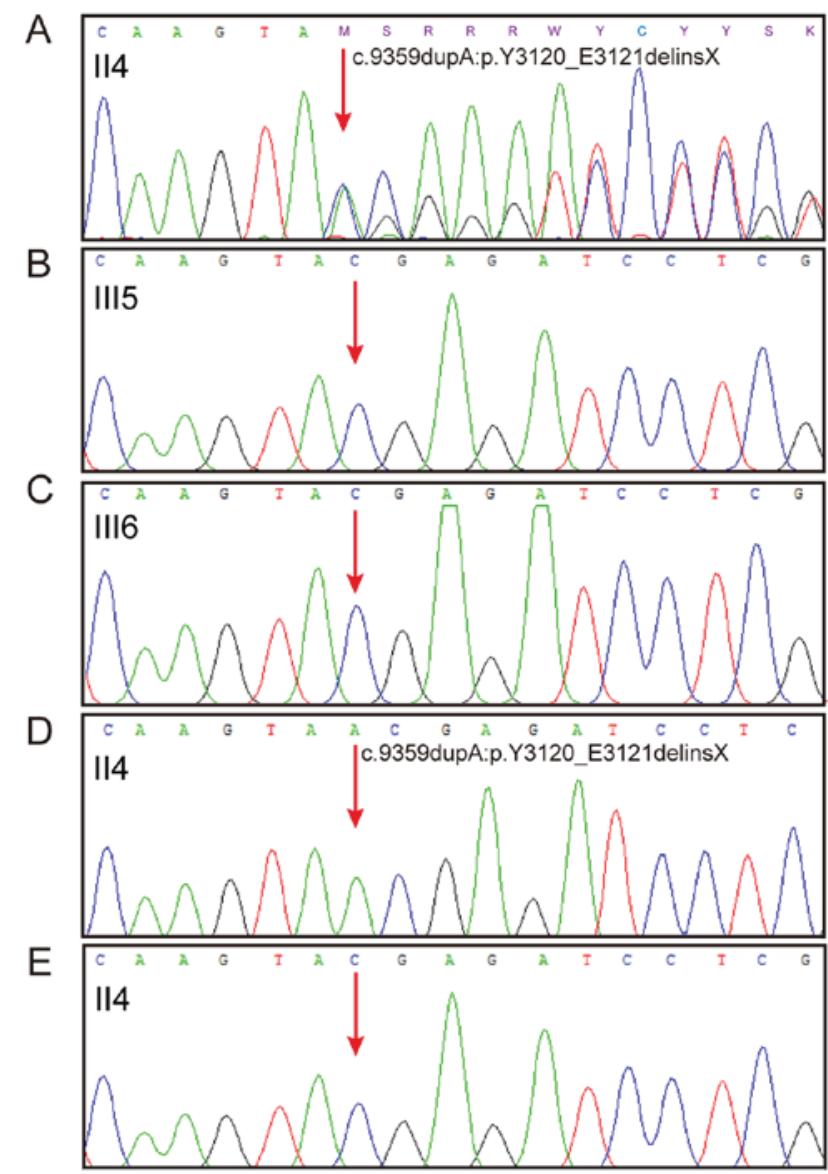

Figure 2. Sanger sequencing results demonstrating the duplication variant c.9359dupA:p.Y3120_E3121delinsX. (A) Nest PCR product from the proband $\left(\mathrm{II}_{4}\right)$. (B) Nest PCR product from the first daughter $\left(\mathrm{III}_{5}\right)$. (C) Nest PCR product from the second daughter $\left(\mathrm{III}_{6}\right)$. (D and E) T-vector ligation product from the proband demonstrating the (D) mutated and (E) wild-type sequences. Arrows indicate the position of the duplication variant or the corresponding wild-type base. PCR, polymerase chain reaction.

According to the domains and repeats divisions listed in UniProt, the frequencies of likely pathogenic substitutions were calculated in the various regions (Table IV). The frequencies in the N-terminal leucine-rich repeat (LRR) domain (LRRNT, amino acids 24-67), LRR domain 1 (LRR1, amino acids 68-91), LRR domain 2 (LRR2, amino acids 92-113), C-terminal LRR domain (LRRCT, amino acids 125-178), cell wall integrity and stress-response component 1 (WSC, amino acids 177-271) and C-type lectin (amino acids 415-531) motifs, which are located in the PC1 C-terminal region, were notably higher. The GPS domain (amino acids 3,012-3,061) appeared to exhibit a relatively high frequency $(11.9 \%, 7$ out of 59 amino acids) compared with the other domains. The c.G9022A:p. V3008M missense mutation is located in an undefined region within amino acids 2,834-3,011. This region presented a frequency of $8.9 \%$ ( 21 out of 235 amino acids), which was higher than the majority of other undefined regions, as well as the average frequency of $6.5 \%$ ( 312 out of 4,805 amino acids).

Collectively, the results indicated that the c.G9022A:p. V3008M missense mutation was likely neutral in PKDB; however, it may be pathogenic. This finding may explain emerging concepts in PKD, including hypomorphic alleles and gene dosage. Further investigations should be performed to identify the potential underlying mechanism.

\section{Discussion}

In the present study, a four-generation ADPKD family including 29 individuals was examined. According to the pedigree, there were five patients with PKD distributed across two generations, including two females $\left(\mathrm{II}_{4}\right.$ and $\left.\mathrm{II}_{10}\right)$ and three males $\left(\mathrm{I}_{1}, \mathrm{II}_{1}\right.$, and $\mathrm{II}_{13}$. A parent of the proband suffered from PKD; however, neither of the daughters were affected. Of the individuals assessed for PKD, almost half (5 patients among 11 individuals with lineal consanguinity) were patients with PKD. Collectively, the results allowed this family to be diagnosed with ADPKD.

Following whole exome sequencing and analysis, the duplication variant NM_001009944.2:c.9359dupA:p. Y3120_E3121delinsX and the missense mutation c.G9022A:p. V3008M in PKD1 can be considered as candidate pathogenic factors accounting for the family's disease. At present, $>2,000$ and $>400$ mutations in PKD1 and PKD2, respectively, have been identified according to PKDB; however, the association between phenotype and genotype requires further study. Dedoussis et al (31) reported that the coexistence of PKD1 and PKD2 mutations in a single patient may induce a more severe phenotype compared with individuals with PKD1 mutations. Numerous reports have demonstrated that patients with PKD1 mutations usually progress to ESRD at an earlier age, particularly those expressing truncated proteins compared with individuals harboring PKD2 mutations (32-34). The novel duplication variant c.9359dupA:p.Y3120_E3121delinsX in PKD1 may contribute to understanding the association between phenotype and genotype in future studies.

PKD1 is a long gene with 46 exons, among which exons 1-35 exhibit six highly similar repeats on chromosome $16(35,36)$. Considering the complexity of PKD1, nest PCR was employed to amplify the candidate fragments. The results of Sanger sequencing for the nest PCR product demonstrated 


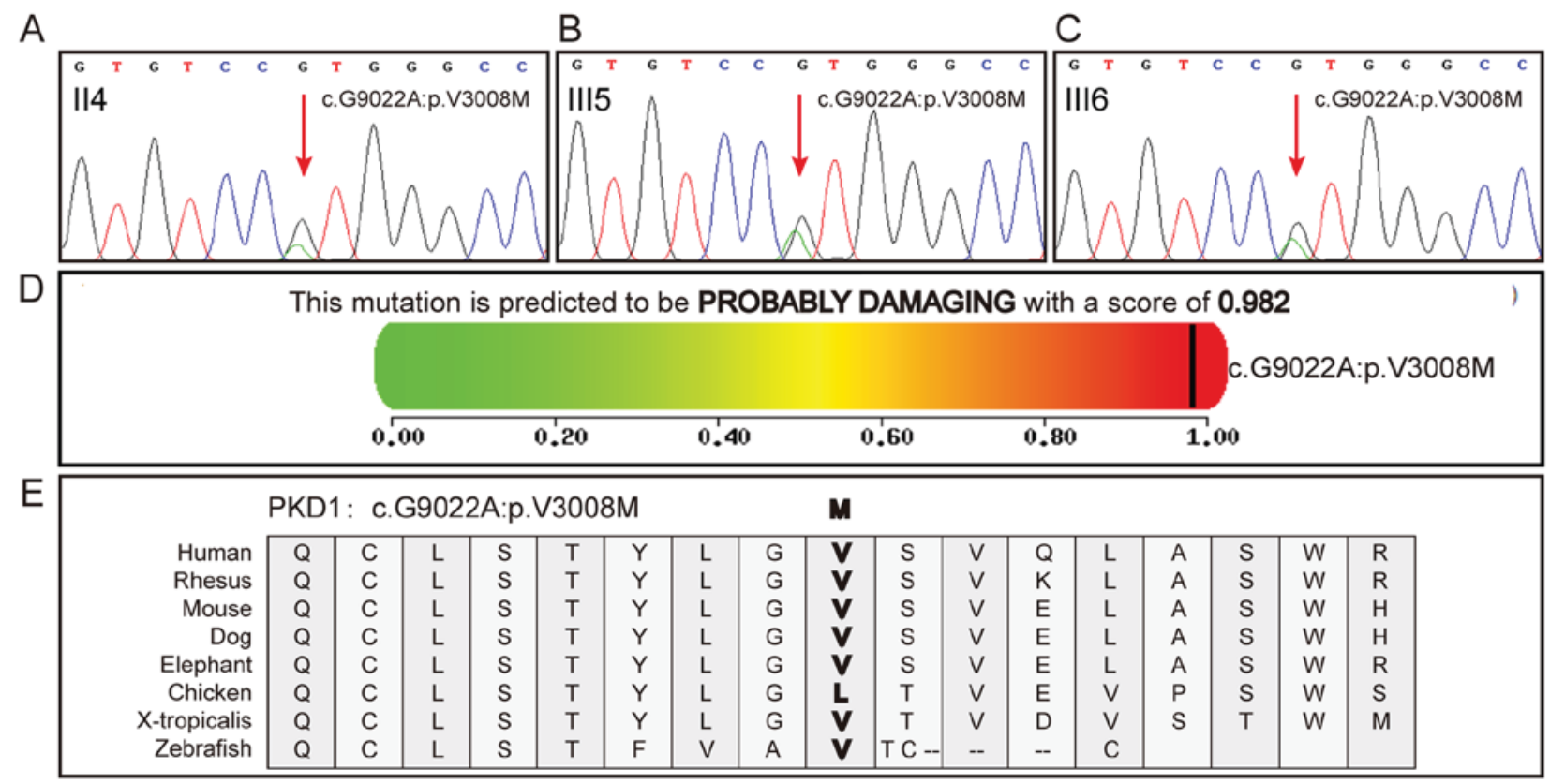

Figure 3. Information for the missense mutation c.G9022A:p.V3008M. (A) Nest PCR product from the proband (II $)$. (B) Nest PCR product from the first daughter $\left(\mathrm{III}_{5}\right.$ ). (C) Nest PCR product from the second daughter (III ${ }_{6}$ ). (D) Pathogenic properties of the missense site, according to the prediction tool Polyphen2_HVAR. (E) Conservation of p.V3008, according to the University of California Santa Cruz Gene Browser. PCR, polymerase chain reaction.

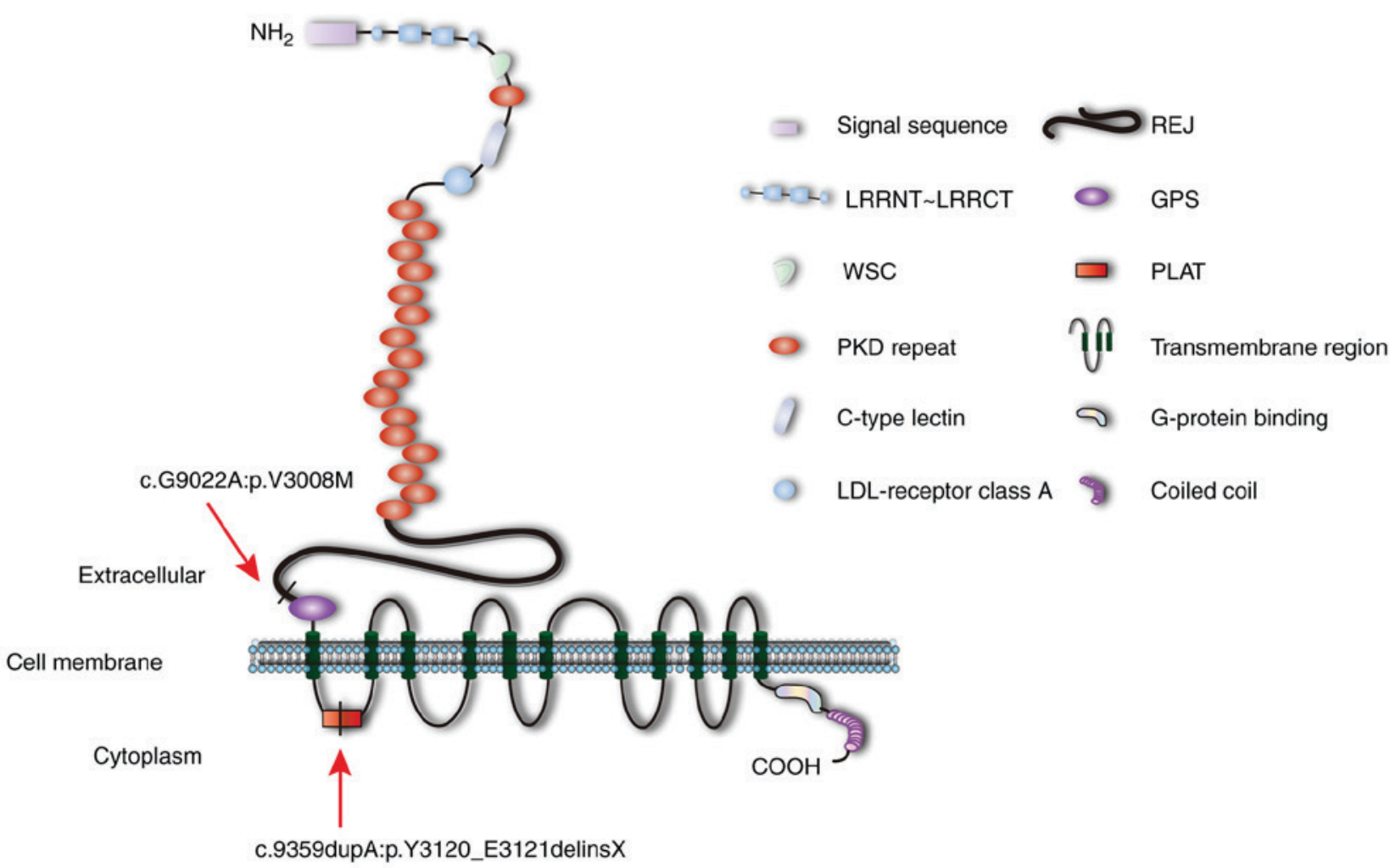

Figure 4. Diagram of polycystin-1. Arrows indicate the position of c.9359dupA:p.Y3120_E3121delinsX within the PLAT domain and c.G9022A:p.V3008M within an undefined region (amino acids 2,834-3,011). GPS, G protein-coupled receptor proteolysis site; LDL, low-density lipoprotein; LRR, leucine-rich repeat; LRRCT, C-terminal LRR domain; LRRNT, N-terminal LRR domain; PKD, polycystic kidney disease; PLAT, polycystin-1, lipoxygenase, $\alpha$-toxin; REJ, receptor egg jelly; WSC, cell wall integrity and stress-response component 1.

that an A was inserted into the coding sequence, resulting in a frameshift mutation. PKDB, OMIM and HGMD were examined, and published articles were employed to determine whether the mutation was novel. There are no previous reports of this duplication variant; however, the missense mutation c.G9361A:p.E3121K, representing the next amino acid substitution of the duplication variant, has been reported to be likely pathogenic (32). Following post-translational modification, 
Table IV. Pathogenic substitutions and domains in PKD1.

\begin{tabular}{|c|c|c|c|c|c|c|c|}
\hline Region & Domain & $\begin{array}{l}\text { Amino acid } \\
\text { position }\end{array}$ & $\begin{array}{l}\text { Amino acid } \\
\text { length (aa) }\end{array}$ & $\begin{array}{l}\text { Nucleotide } \\
\text { length (bp) }\end{array}$ & $\begin{array}{l}\text { Number } \\
\text { of SNP }\end{array}$ & $\begin{array}{c}\text { Number of } \\
\text { pathogenic SNPs }\end{array}$ & $\begin{array}{c}\text { Pathogenic SNPs/total } \\
\text { SNPs }(\%)\end{array}$ \\
\hline \multirow[t]{39}{*}{ Extracellular } & LRRNT & $24-67$ & 44 & 132 & 24 & 4 & 16.7 \\
\hline & LRR1 & $68-91$ & 24 & 72 & 18 & 4 & 22.2 \\
\hline & LRR2 & $92-113$ & 22 & 66 & 22 & 5 & 22.7 \\
\hline & UD & $114-124$ & 11 & 33 & 8 & 1 & 12.5 \\
\hline & LRRCT & $125-178$ & 54 & 162 & 26 & 4 & 15.4 \\
\hline & WSC & $177-271$ & 95 & 285 & 63 & 9 & 14.3 \\
\hline & PKD1 & $272-359$ & 88 & 264 & 73 & 4 & 5.5 \\
\hline & UD & $360-414$ & 55 & 165 & 38 & 3 & 7.9 \\
\hline & C-type lectin & $415-531$ & 117 & 351 & 95 & 18 & 18.9 \\
\hline & UD & $532-637$ & 106 & 318 & 83 & 5 & 6 \\
\hline & $\begin{array}{l}\text { LDL-receptor } \\
\text { class A }\end{array}$ & $638-671$ & 34 & 102 & 37 & 1 & 2.7 \\
\hline & UD & $672-742$ & 71 & 213 & 80 & 7 & 8.8 \\
\hline & PKD2 & $743-817$ & 34 & 102 & 60 & 1 & 1.7 \\
\hline & UD & $818-854$ & 37 & 111 & 43 & 1 & 2.3 \\
\hline & PKD3 & $855-928$ & 75 & 225 & 83 & 0 & 0 \\
\hline & UD & $929-934$ & 6 & 18 & 2 & 1 & 50 \\
\hline & PKD4 & $935-1020$ & 74 & 222 & 80 & 4 & 5 \\
\hline & UD & $1021-1022$ & 2 & 6 & 1 & 0 & 0 \\
\hline & PKD5 & $1023-1129$ & 86 & 258 & 134 & 1 & 0.7 \\
\hline & PKD6 & $1127-1215$ & 107 & 321 & 112 & 4 & 3.6 \\
\hline & PKD7 & $1213-1298$ & 86 & 258 & 120 & 5 & 4.2 \\
\hline & PKD8 & 1294-1383 & 90 & 270 & 135 & 5 & 3.7 \\
\hline & PKD9 & $1382-1469$ & 88 & 264 & 101 & 4 & 4 \\
\hline & PKD10 & $1468-1551$ & 84 & 252 & 98 & 6 & 6.1 \\
\hline & PKD11 & $1550-1635$ & 86 & 258 & 110 & 1 & 0.9 \\
\hline & PKD12 & $1634-1721$ & 88 & 264 & 110 & 3 & 2.7 \\
\hline & PKD13 & 1719-1805 & 87 & 261 & 109 & 1 & 0.9 \\
\hline & UD & 1806 & 1 & 3 & 2 & 0 & 0 \\
\hline & PKD14 & $1807-1890$ & 84 & 252 & 110 & 6 & 5.5 \\
\hline & PKD15 & 1889-1974 & 86 & 258 & 107 & 2 & 1.9 \\
\hline & UD & 1976 & 1 & 3 & 2 & 0 & 0 \\
\hline & PKD16 & $1977-2057$ & 81 & 243 & 116 & 8 & 6.9 \\
\hline & UD & 2058-2059 & 2 & 6 & 9 & 0 & 0 \\
\hline & PKD17 & $2060-2148$ & 89 & 267 & 103 & 3 & 2.9 \\
\hline & REJ & $2146-2833$ & 688 & 2064 & 863 & 67 & 7.8 \\
\hline & UD & 2834-3011 & 178 & 534 & 235 & 21 & 8.9 \\
\hline & GPS & $3012-3061$ & 50 & 150 & 59 & 7 & 11.9 \\
\hline & UD & $3062-3074$ & 13 & 39 & 15 & 1 & 6.7 \\
\hline & & 24-3074 & 3051 & 9153 & 3444 & 216 & 6.3 \\
\hline \multirow[t]{4}{*}{ Transmembrane } & UD & $3075-3117$ & 43 & 129 & 40 & 3 & 7.5 \\
\hline & PLAT & $3118-3233$ & 116 & 348 & 113 & 5 & 4.4 \\
\hline & UD & $3234-4106$ & 873 & 2619 & 958 & 80 & 8.4 \\
\hline & & $3075-4106$ & 1032 & 3096 & 1111 & 88 & 7.9 \\
\hline \multirow[t]{2}{*}{ Cytoplasmic } & UD & $4107-4303$ & 197 & 591 & 250 & 8 & 3.2 \\
\hline & UD & $4107-4303$ & 197 & 591 & 250 & 8 & 3.2 \\
\hline Total & & 24-4303 & 4280 & 12840 & 4805 & 312 & 6.5 \\
\hline
\end{tabular}

Bp, base pair; GPS, G protein-coupled receptor proteolysis site; LRR, leucine-rich repeat; LDL, low density lipoprotein; LRR1, LRR domain 1; LRR2, LRR domain 2; LRRCT, C-terminal LRR domain; LRRNT, N-terminal LRR domain; PKD, polycystic kidney disease; PLAT, polycystin-1, lipoxygenase, $\alpha$-toxin; REJ, receptor egg jelly; SNP, single nucleotide polymorphism; UD, undefined region; WSC, cell wall integrity and stress-response component 1. 
PC1 constitutes 4,279 amino acids, with numerous functional domains. The c.9359dupA:p.Y3120_E3121delinsX duplication variant altered a TAC codon to TAA, which is a termination signal in protein translation. As a result, protein translation was stopped at the 3,120th amino acid (tyrosine, T), and a truncated $\mathrm{PC} 1$ protein was generated. The truncated PC1 was terminated in the PLAT domain, which may have affected cell signaling mediated by the protein and its roles as a lipid/protein binding scaffold (19). The premature stop codon resulted in an $\sim 3,000$ amino acid PC1 sequence, causing the protein to lose the majority of its C-terminal domains.

A missense site (NM_001009944.2:c.G9022A:p.V3008M) and two synonymous sites (NM_001009944.2:c.G4674A:p. T1558T and NM_001009944.2: c.G3444A:p.P1148P) in PKD1 were also observed according to the whole exome sequencing results from the proband. All three substitutions were reported as likely neutral in PKDB.

The missense site c.G9022A:p.V3008M is located in the 25th exon of PKD1, which lies in an undefined region (amino acids 2,834-3,011) near the GPS motif, upstream of c.9359dupA:p.Y3120_E3121delinsX. The 9,022nd nucleotide (guanine, G) in the consensus coding sequence containing 12,912 nucleotides is changed to A, resulting in an amino acid substitution from $\mathrm{V}$ to methionine (M). $\mathrm{V}$ is a nonpolar amino acid, while $\mathrm{M}$ is polar, indicating that a structural alteration occurs in the PKD1 protein PC1 following substitution. When pathogenic properties were assessed with the prediction tools, the missense mutation (c.G9022A:p.V3008M) was indicated as likely to lead to a diseased state. The results of conservation analysis also supported the pathogenic properties of the missense mutation. Additionally, the frequency of pathogenic substitutions in various regions was analyzed. The region (from amino acids 2,834-3,011) that contained the missense mutation (c.G9022A:p.V3008M) exhibited a relatively high frequency. Collectively, the results indicated that the c.G9022A:p.V3008M missense mutation is likely to be pathogenic; however, it was identified as likely neutral in PKDB.

The results regarding pathogenic substitutions in PKD1 within various regions also revealed that the PC1 N-terminal domain, including LRRNT, LRR1, LRR2, LRRCT, WSC and $\mathrm{C}$-type lectin, exhibited a relatively high frequency compared with the C-terminal domain, suggesting that missense mutations in the N-terminus are more likely to induce ADPKD. Additionally, the frequency of pathogenic substitutions in the C-type lectin domain was notably higher, indicating that it is a key functional domain.

To determine whether the missense mutation is a disease-inducing factor along with the duplication variant, Sanger sequencing was employed to detect the missense mutation in the proband and their two normal daughters. The missense mutation was observed in the sequencing results for all three individuals, indicating that the missense mutation was not a pathogenic factor in the family with ADPKD. It should be noted that the c.G9022A:p.V3008M missense mutation may not be responsible for PKD in the studied family; however, it may be involved in the process of PKD development according to the gene dosage theory (20).

The present study identified a novel duplication variant, NM_001009944.2:c.9359dupA:p.Y3120_E3121delinsX,within the PLAT domain, and a missense mutation, c.G9022A:p.
V3008M in PKD1, within an undefined region (amino acids 2,834-3,011), in a family that suffers from ADPKD. The duplication variant is considered to be the pathogenic factor for ADPKD in this family; however, the c.G9022A:p.V3008M missense mutation has an uncertain importance. Numerous, but not all, classical methods were used in the study to assess the pathogenic properties of missense mutations. Only three individuals were sequenced, as informed consent was not obtained from other individuals in the family; the assessment of additional samples may improve understanding into the genetic basis of PKD. Furthermore, recently reported novel substitutions were not included in the calculation of pathogenic mutations within various regions in PC1 (37-39). The pathogenic mechanism of the variants, as well as the association with clinical manifestations, require further investigation.

\section{Acknowledgements}

The authors would like to thank Dr Xue Zhang (Institute of Basic Medical Sciences of the Chinese Academy of Medical Sciences, McKusick-Zhang Center for Genetic Medicine, School of Basic Medicine, Harbin Medical University, Harbin , China) for his advice and help. The authors also thank Dr Mengdi Cai (Laboratory of Medical Genetics, Harbin Medical University, Harbin, China) for the modification of Fig. 4.

\section{Funding}

The present study was supported by the National Key Research and Development Program of China (grant no. 2016YFC1000504).

\section{Availability of data and materials}

The datasets used and/or analyzed during the current study are available from the corresponding author on reasonable request.

\section{Authors' contributions}

KD, XJ, JW, WS, JY, XZ and SF conceived and designed the study; HM, HWu, JS, HWa and XZ collected the clinical information; KD, HM, HWu, JS, WJ, HS, SZ and GJ performed the experiments; KD, LX, XZ, GJ, RG and JB analyzed the data; and KD, HWa, WS and SF prepared the manuscript. All authors read and approved the final manuscript.

\section{Ethics approval and consent to participate}

The present study was approved by the Institutional Research Board of Harbin Medical University (no. HMUIRB20180001, Harbin, China), and all participants provided written informed consent.

\section{Patient consent for publication}

Not applicable.

\section{Competing interests}

The authors declare that they have no competing interests. 


\section{References}

1. Obeidova L, Elisakova V, Stekrova J, Reiterova J, Merta M, Tesar V, Losan F and Kohoutova M: Novel mutations of PKD genes in the Czech population with autosomal dominant polycystic kidney disease. BMC Med Genet 15: 41, 2014.

2. Edrees BM, Athar M, Abduljaleel Z, Al-Allaf FA, Taher MM, Khan W, Bouazzaoui A, Al-Harbi N, Safar R, Al-Edressi H, et al: Functional alterations due to amino acid changes and evolutionary comparative analysis of ARPKD and ADPKD genes. Genom Data 10: 127-134, 2016.

3. Robinson C, Hiemstra TF, Spencer D, Waller S, Daboo L, Karet Frank1 FE and Sandford RN: Clinical utility of PKD2 mutation testing in a polycystic kidney disease cohort attending a specialist nephrology out-patient clinic. BMC Nephrol 13: 79, 2012.

4. Abdelwahed M, Hilbert P, Ahmed A, Mahfoudh H, Bouomrani S, Dey M, Hachicha J, Kamoun H, Keskes-Ammar L and Belguith N: Mutational analysis in patients with Autosomal Dominant Polycystic Kidney Disease (ADPKD): Identification of five mutations in the PKD1 gene. Gene: May 31, 2018 (Epub ahead of print)

5. Yu G, Qian X, Wu Y, Li X, Chen J, Xu J and Qi J: Analysis of gene mutations in PKD1/PKD2 by multiplex ligation-dependent probe amplification: Some new findings. Ren Fail 37: 366-371, 2015.

6. Litvinchuk T, Tao Y, Singh R and Vasylyeva TL: A case of new familiar genetic variant of autosomal dominant polycystic kidney disease-2: A case study. Front Pediatr 3: 82, 2015.

7. Casteleijn NF, Spithoven EM, Rookmaaker MB, Vergouwen MD and Gansevoort RT: Bilateral cysts in the choroid plexus in a patient with autosomal dominant polycystic kidney disease. Nephrol Dial Transplant 30: 859-860, 2015.

8. Liu B, Chen SC, Yang YM, Yan K, Qian YQ, Zhang JY, Hu YT, Dong MY, Jin F, Huang HF and Xu CM: Identification of novel PKD1 and PKD2 mutations in a Chinese population with autosomal dominant polycystic kidney disease. Sci Rep 5: 17468, 2015

9. Hafer AS and Conran RM: Autosomal recessive polycystic kidney disease. Acad Pathol 4: 2374289517718560, 2017.

10. Thomas C, Zühlsdorf A, Hörtnagel K, Mulahasanovic L, Grauer OM, Kümpers P, Wiendl H and Meuth SG: A novel PKD1 mutation associated with autosomal dominant kidney disease and cerebral cavernous malformation. Front Neurol 9: 383, 2018.

11. Somlo S, Wirth B, Germino GG, Weinstat-Saslow D, Gillespie GA, Himmelbauer H, Steevens L, Coucke P, Willems P, Bachner L, et al: Fine genetic localization of the gene for autosomal dominant polycystic kidney disease (PKD1) with respect to physically mapped markers. Genomics 13: 152-158, 1992.

12. Gainullin VG, Hopp K, Ward CJ, Hommerding CJ and Harris PC: Polycystin-1 maturation requires polycystin-2 in a dose-dependent manner. J Clin Invest 125: 607-620, 2015.

13. Kim DY and Park JH: Genetic mechanisms of ADPKD. Adv Exp Med Biol 933: 13-22, 2016.

14. Shin YB and Park JH: Recent trends in ADPKD research. Adv Exp Med Biol 933: 3-11, 2016.

15. Yang $Y$ and Ehrlich BE: Structural studies of the C-terminal tail of polycystin-2 (PC2) reveal insights into the mechanisms used for the functional regulation of PC2. J Physiol 594: 4141-4149, 2016.

16. Venugopal $\mathrm{J}$ and Blanco G: On the many actions of ouabain: Pro-cystogenic effects in autosomal dominant polycystic kidney disease. Molecules 22: E729, 2017.

17. Rangan GK, Lopez-Vargas P, Nankivell BJ, Tchan M, Tong A, Tunnicliffe DJ and Savige J: Autosomal dominant polycystic kidney disease: A path forward. Semin Nephrol 35: 524-537, 2015

18. Saigusa T and Bell PD: Molecular pathways and therapies in autosomal-dominant polycystic kidney disease. Physiology (Bethesda) 30: 195-207, 2015.

19. Ong AC and Harris PC: A polycystin-centric view of cyst formation and disease: The polycystins revisited. Kidney Int 88: 699-710, 2015.

20. Santoso NG, Cebotaru L and Guggino WB: Polycystin-1, 2, and STIM1 interact with IP(3)R to modulate ER Ca release through the PI3K/Akt pathway. Cell Physiol Biochem 27: 715-726, 2011

21. Yamaguchi T, Wallace DP, Magenheimer BS, Hempson SJ, Grantham JJ and Calvet JP: Calcium restriction allows cAMP activation of the B-Raf/ERK pathway, switching cells to a cAMP-dependent growth-stimulated phenotype. J Biol Chem 279: 40419-40430, 2004.
22. Yamaguchi T, Pelling JC, Ramaswamy NT, Eppler JW, Wallace DP, Nagao S, Rome LA, Sullivan LP and Grantham JJ: cAMP stimulates the in vitro proliferation of renal cyst epithelial cells by activating the extracellular signal-regulated kinase pathway. Kidney Int 57: 1460-1471, 2000.

23. Rossetti S, Kubly VJ, Consugar MB, Hopp K, Roy S, Horsley SW, Chauveau D, Rees L, Barratt TM, van't Hoff WG, et al: Incompletely penetrant PKD1 alleles suggest a role for gene dosage in cyst initiation in polycystic kidney disease. Kidney Int 75: 848-855, 2009.

24. Nagao S, Nishii K, Yoshihara D, Kurahashi H, Nagaoka K, Yamashita T, Takahashi H, Yamaguchi T, Calvet JP and Wallace DP: Calcium channel inhibition accelerates polycystic kidney disease progression in the $\mathrm{Cy} /+$ rat. Kidney Int 73 : 269-277, 2008

25. Ghata $\mathbf{J}$ and Cowley BD Jr: Polycystic kidney disease. Compr Physiol 7: 945-975, 2017.

26. Trudel M, Yao Q and Qian F: The role of G-protein-coupled receptor proteolysis site cleavage of polycystin-1 in renal physiology and polycystic kidney disease. Cells 5: E3, 2016.

27. Chapin HC, Rajendran V and Caplan MJ: Polycystin-1 surface localization is stimulated by polycystin- 2 and cleavage at the $\mathrm{G}$ protein-coupled receptor proteolytic site. Mol Biol Cell 21: 4338-4348, 2010

28. Li H, Handsaker B, Wysoker A, Fennell T, Ruan J, Homer N, Marth G, Abecasis G and Durbin R; 1000 Genome Project Data Processing Subgroup: The Sequence Alignment/Map format and SAMtools. Bioinformatics 25: 2078-2079, 2009.

29. Al-Bhalal L and Akhtar M: Molecular basis of autosomal dominant polycystic kidney disease. Adv Anat Pathol 12: 126-133, 2005.

30. Gout AM, Martin NC, Brown AF and Ravine D: PKDB: Polycystic kidney disease mutation database-a gene variant database for autosomal dominant polycystic kidney disease. Hum Mutat 28: 654-659, 2007.

31. Dedoussis GV, Luo Y, Starremans P, Rossetti S, Ramos AJ, Cantiello HF, Katsareli E, Ziroyannis P, Lamnissou K, Harris PC and Zhou J: Co-inheritance of a PKD1 mutation and homozygous PKD2 variant: A potential modifier in autosomal dominant polycystic kidney disease. Eur J Clin Invest 38: 180-190, 2008.

32. Cornec-Le Gall E, Audrézet MP, Chen JM, Hourmant M, Morin MP, Perrichot R, Charasse C, Whebe B, Renaudineau E, Jousset P, et al: Type of PKD1 mutation influences renal outcome in ADPKD. J Am Soc Nephrol 24: 1006-1013, 2013.

33. Neumann HP, Bacher J, Nabulsi Z, Ortiz Brüchle N, Hoffmann MM, Schaeffner E, Nürnberger J, Cybulla M, Wilpert J, Riegler P, et al: Adult patients with sporadic polycystic kidney disease: The importance of screening for mutations in the PKD1 and PKD2 genes. Int Urol Nephrol 44: 1753-1762, 2012.

34. Yu C, Yang Y, Zou L, Hu Z, Li J, Liu Y, Ma Y, Ma M, Su D and Zhang S: Identification of novel mutations in Chinese Hans with autosomal dominant polycystic kidney disease. BMC Med Genet 12: 164, 2011.

35. Kinoshita M, Higashihara E, Kawano H, Higashiyama R, Koga D, Fukui T, Gondo N, Oka T, Kawahara K, Rigo K, et al: Technical evaluation: Identification of pathogenic mutations in PKD1 and PKD2 in patients with autosomal dominant polycystic kidney disease by next-generation sequencing and use of a comprehensive new classification system. PLoS One 11: e0166288, 2016.

36. Liu J, Li L and Liu Q: Mutational analysis of PKD1 gene in a Chinese family with autosomal dominant polycystic kidney disease. Int J Clin Exp Pathol 8: 13289-13292, 2015.

37. Carrera P, Calzavara S, Magistroni R, den Dunnen JT, Rigo F, Stenirri S, Testa F, Messa P, Cerutti R, Scolari F, et al: Deciphering variability of PKD1 and PKD2 in an italian cohort of 643 patients with autosomal dominant polycystic kidney disease (ADPKD). Sci Rep 6: 30850, 2016.

38. Sha YK, Sha YW, Mei LB, Huang XJ, Wang X, Lin SB, Li L and Li P: Use of targeted sequence capture and high-throughput sequencing identifies a novel PKD1 mutation involved in adult polycystic kidney disease. Gene 634: 1-4, 2017.

39. Mallawaarachchi AC, Furlong TJ, Shine J, Harris PC and Cowley MJ: Population data improves variant interpretation in autosomal dominant polycystic kidney disease. Genet Med: Oct29,2018(Epubaheadofprint).doi: 10.1038/s41436-018-0324-x.

This work is licensed under a Creative Commons Attribution-NonCommercial-NoDerivatives 4.0 International (CC BY-NC-ND 4.0) License. 